\title{
PELEANDO A LA CONTRA: CHARLES BUKOWSKI Y LA POSICIÓN DEL REALISMO SUCIO EN LA CONTRACULTURA DEL SIGLO XX
}

\author{
Manuel Mata Piñeiro \\ Universidad de Vigo. Departamento de Pintura. Grupo de investigación "Arte e Contextos".
}

\section{Resumen}

El artículo gira en torno a la figura de Charles Bukowski como uno de los máximos exponentes del movimiento literario denominado "realismo sucio", y de cómo dicho movimiento constituyó su posición, mediante una serie de fundamentos que necesariamente han ejercido una fuerte influencia en numerosos ámbitos, en la contracultura del siglo XX, conformando una opción marginal, periférica y alternativa a las ramas de pensamiento más populares asociadas a la misma.

Palabras clave: BUKOWSKI, CHARLES; REALISMO SUCIO; CONTRACULTURA MARGINAL.

\section{FIGHTING AGAINST: CHARLES BUKOWSKI AND THE POSITION OF DIRTY \\ REALISM IN THE TWENTIETH CENTURY COUNTERCULTURE.}

Abstract

The article revolves around the figure of Charles Bukowski as one of the greatest exponents of the literary movement called "dirty realism", and how that movement constituted its position, through a series of foundations that necessarily have exerted a strong influence in many areas, In the counterculture of the twentieth century, forming a marginal, peripheral and alternative to the most popular branches of thought associated with it.

Keywords: BUKOWSKI, CHARLES; DIRTY REALISM; MARGINAL COUNTERCULTURE

\footnotetext{
Mata Piñeiro, Manuel. 2017 . "Peleando a la contra: Charles Bukowski y la posición del realismo sucio en la contracultura del siglo XX". AusArt 5 (1): 169-177 D0I: 10.1387/ausart.17465
}

\section{AUSART}




\section{INTRODUCCIÓN}

Situándonos en los Estados Unidos de la segunda mitad del siglo pasado, parece obligado en cualquier individuo el hecho de situarse en uno de los dos bandos mayoritarios, definidos por las grandes ideologías que bullían en la sociedad del momento. Por un lado, tenemos al sector más conservador, a la autoridad gubernamental, los partidarios de la guerra de Vietnam, los censores, los protectores de la moral estadounidense. Por otro, opuestos al primer grupo, los denominados hippies, los marginados, los exaltadores, los pacifistas. En resumen, todo el sector social que difería ideológica, sexual, intelectual o políticamente de los primeros.

A bote pronto, esa es la imagen elemental de los Estados Unidos que suele visualizarse cuando uno quiere remontarse a la intensa década de los sesenta, pero basta con ahondar un poco en la superficie para comprender que existía una mayor diversidad de pensamiento de la que un par de frases puedan abarcar. Una de estas corrientes periféricas, veladas por la despampanante fama de Jimmy Hendrix, el festival Woodstock, el boom beat de los sesenta, la guerra de Vietnam y demás, es el movimiento literario denominado popularmente como realismo sucio.

\section{REALISMO SUCIO}

Si bien otros autores tuvieron una denotada relevancia en el movimiento, como John Fante (1909-1983) y Raymond Carver (1938-1988), fue Charles Bukowski (1920-1994) quien acumuló un mayor público y postuló más nítidamente los fundamentos del movimiento mediante una extensa obra que fluctuó desde la poesía al ensayo, pasando por el relato, la novela y el guión cinematográfico. Consolidado por su excelente mala fama, Charles Bukowski ha pasado a la historia como un "escritor maldito". De pedir a una decena de lectores que nos explicasen de qué habla la poesía de Bukowski, pronto oiríamos una sucesión de temas repetidos: alcohol, juego, mujeres, prostitutas, más alcohol, borrachos, sexo, juego y, quizá, gatos y escritura. No obstante, es necesario apartar el mito que se ha creado en torno a la figura del autor, y concentrar el análisis en lo que pretendía decir. 
No defender una postura o ideología mayoritaria, ya sea de carácter autoritario o subversivo, confiere al individuo el aspecto marginal y nihilista que termina por excluirlo del panorama socio-cultural. Este fue el caso de Bukowski.

Un individuo que no milita en una revolución y asimismo se niega a luchar en su contra es, desde el punto de vista de ambos bandos, el enemigo. De modo que, necesariamente, se consolida en torno al doble desertor un singular aislamiento.

\subsection{Sin heroísmos}

bueno, la mayoría tenemos

héroes

y no queremos que

sean corrientes,

queremos que sean arriesgados

y originales a no poder más

y que nunca hagan

la más mínima

concesión de ninguna clase.

“La muerte de un héroe" (Bukowski [2002] 2005)

Uno de los principales fundamentos del realismo sucio es la ausencia de héroe. El protagonista se desentiende de las grandes gestas, sea cual sea su naturaleza, y se centra, en última instancia, en sí mismo. Ello no implica que la personalidad del autor haya de ser mesurada o complaciente. Nada más lejos, pues, dado que no es necesario rendir cuentas a ninguna consigna, doctrina o institución con grandes actos significativos, el autor conquista un particular rincón de libertad donde la única preocupación es afrontar su propia idiosincrasia y experimentar su intransferible estilo de vida. Para comprender en condiciones la esencia de este antihéroe, de cómo el rechazo de heroísmos es utilizado como herramienta, es necesario un establecer un contrapunto.

Pensemos en un autor situado en el sector social más subversivo, coetáneo a Charles Bukowski y que, además, cuente también con una actitud reconocidamente marginal: Allen Ginsberg, cabecilla de la beat generation. Si bien muchos de los temas tocados por Bukowski están presentes en la obra de Ginsberg, existe una distinción clave a la hora de analizar las diferencias en sus respectivas posturas. Dicho elemento no es otro que la representación. 
En Howl, el poema que sirvió de pistoletazo de salida a la beat generation, el tema esencial es la reivindicación de un estilo de vida, de la existencia de todo un abanico de ciudadanos cuyos actos, mal vistos por la sociedad, merecen reconocimiento. Ginsberg se convierte, tras su publicación, en portavoz de los marginados, de la libertad considerada tabú. No hay que olvidar cómo comienza el mentado poema ( $/$ saw the best minds of my generation destroyed by madness), pues desde un primer momento la obra es configurada como un testimonio debido y en homenaje a terceros, elaborado mediante un conjunto de experiencias provenientes de distintos individuos.

Bukowski, en el otro extremo, centra su discurso en una sencilla primera persona del singular. Habla de sí mismo y de lo que, a su manera de ver las cosas, le concierne. Se distancia de la mitología y el dramatismo en favor de su propia identidad. Es decir, es portavoz exclusivamente de sí mismo. Dicha exclusividad es la causante, asimismo, de que las analogías en donde participan grandes símbolos de la cultura popular sean empleadas de manera diametralmente opuesta por cada uno de los autores. Ginsberg, por su parte, utiliza estos símbolos para conferir una cualidad superior a aquello de lo que habla. Es decir, hace que los drogadictos, por ejemplo, asciendan hasta la categoría de ángeles. Bukowski, sin embargo, ejerce el viaje contrario. De modo que si habla del infierno, por ejemplo, hace que sea el infierno el que descienda hasta su cotidianidad y se encarne en algo mundano. Es decir, Bukowski debilita los símbolos, imponiéndoles una mortalidad común; Ginsberg se apropia de ellos, infundiéndoles una identidad concreta, sin que por el camino desaparezca el sentido épico que poseen.

En definitiva, Ginsberg, posicionado en uno de los bandos, se erige como un superviviente del estilo de vida que defiende y un representante de su generación, mientras que, Bukowski, al elaborar una perspectiva discursiva imposible de trasladar o extrapolar, eliminando concienzudamente los puntos en común con su entorno, se convierte en el antihéroe por excelencia. Ahora, habría que considerar cuál de estas posturas fue, en lo que al ámbito cultural respecta, la más necesaria.

\section{EL LEGADO DE WHITMAN}

Recordemos que Walt Whitman, escritor del aclamado Leaves of grass, considerado padre del verso libre e introductor de la primera persona narrativa 
en la poesía estadounidense, propuso en su obra, especialmente en Song of myself, un héroe colectivo en contraposición al héroe épico característico de la literatura. Es decir, se centró en que el protagonista de su poesía fuese la gente común, y su método para ello fue enamorarse de cada identidad y adoptar cada doctrina como propia. No descartó nada y trató de reunir el grueso de la naturaleza y el hombre en una sola personalidad. Whitman se convirtió en el ideal de poeta estadounidense y no fueron pocos autores, a su muerte, los que intentaron ocupar el puesto vacante, haciendo apología de todo, exaltando cada pensamiento. En este caso nos encontramos, por ejemplo, con Pablo Neruda, especialmente con su poemario Odas elementales.

Pero si bien es comprensible este intento de sustitución, lo que cabría preguntarse es si dicha sustitución era necesaria. Tal como yo lo veo, lo necesario era retomar el discurso de Whitman con un aporte evolutivo, no insistir en la misma dinámica. Y, considerando que escritores como Neruda no eran el siguiente paso coherente en el camino, me inclino a centrar la mirada en Bukowski y Ginsberg. Ambos autores recogieron el testigo tendido por Whitman, más cada uno echó a correr en una dirección distinta.

Por su parte, Ginsberg sin duda adoptó la actitud predispuesta a las interconexiones, a la solidaridad con sus coetáneos. De manera que el héroe colectivo propuesto por Whitman prevalece en su obra, con la matización de que ahora se trata de un héroe épico colectivo. Las intenciones claramente descritas en poemas como I Hear America Singing o Passage to India, son retomadas por el autor de Howl con el tono necesario para inscribirlas en su propio contexto. Asimismo, en cuanto a estructura, es clara la influencia de Whitman si sometemos a examen la de poemas de Ginsberg, como Footnote to Howl o Psalm III, y los comparamos con, por ejemplo, Starting from Paumanok, de Whitman. Queda patente en ambos una preocupación por la musicalidad y la cadencia que, sin bien conduce de Whitman a Ginsberg, también conecta a Ginsberg con Bob Dylan y, con él, se esclarece la relación del escritor con la cultura popular del momento.

Bukowski, en cambio, continúa con el legado de Whitman no por adscribirse a su admiración por al humanidad, ni por ensalzar la belleza de su país y, mucho menos, por su amor a la harmonía de un discurso. Sino para desarrollar lo indicado por Whitman en el comienzo del poema One's-self I sing: Canto el yo, persona simple, separada ([1855] 2009). La obra de Bukowski está centrada en su propia vida, y su conexión con el espectador se debe precisamente a ello. Al adoptar la soledad y los actos cotidianos como temas fundamentales, si bien la percepción es única, las situaciones son comunes a todo individuo. 
De modo que Bukowski se convierte en heredero evolutivo de Whitman al lograr configurar un discurso donde cualquiera, en mayor o menor medida, puede reconocerse.

La diferencia entre él y Ginsberg es fácil de ejemplificar. Imaginemos a una mujer de cincuenta años a la que, en los años sesenta, por su educación o su estilo de vida, le es imposible conectar con el sentido de Howl, y, sin embargo, sí puede reconocerse en el hombre tendido en la cama, mirando al techo, de un poema de Bukowski. Esta mujer, claro, tendría las mismas reservas hacia la homosexualidad y consumo de peyote del primero, como hacia el alcoholismo empedernido y promiscuidad del segundo. No obstante, el punto clave de la cuestión es que tanto esa mujer, como Ginsberg, los jóvenes protagonistas de Howl o los combatientes de Vietnam, se han encontrado en un escenario similar y propio. Bukowski, al demostrar, voluntaria o involuntariamente, los puntos en común de la humanidad mediante sus propias experiencias, se erige como un heredero consecuente del héroe colectivo de Whitman. Reduciendo la pluralidad a un único individuo y evitando cualquier trasfondo épico. Ahora bien, retomando la posición del realismo sucio en la segunda mitad del siglo XX, y asumiendo a Bukowski como paradigma del movimiento, cabría preguntarse qué fuerzas ejerció, o pretendió ejercer, y qué sentido tenían en su momento.

\title{
3. UN LUGAR APARTADO
}

\author{
¿a qué viene tanto \\ absurdo \\ pavoneo y afectación? \\ ¿por qué arropamos todo lo que decimos \\ con un énfasis especial \\ cuando lo único que hace falta \\ es limitarse a decir \\ aquello que debe decirse?
}

"La muerte de un héroe" (Bukowski [2002] 2005)

El doble sentido en un discurso, se trate de un poema, una pintura o una campaña publicitaria, supone un rodeo, una veladura. A lo largo del siglo XX se ha desarrollado una indudable predisposición a presentar las ideas mediante un retorcido uso de la relación entre significantes y significa- 
dos. De tal manera que, asimilada la representación mental, el mensaje, el meollo de la cuestión, es introducido en los oyentes como una agresiva píldora azucarada. Al más puro estilo Mary Poppins. Incluso Bob Dylan se las ingeniaba para que una denuncia social sonase entretenida. Esta dinámica era, para los autores del realismo sucio y en especial para Charles Bukowski, una espina clavada entre las muelas. Lejos de metáforas, dobles sentidos, analogías suspicaces o semiótica de cualquier índole, el realismo sucio configuró su actitud abogando por un lenguaje sin tapujos. No se trata tanto de ser ofensivo al utilizar vocablos malsonantes, sino de que esos vocablos existen para exponer una realidad determinada con exactitud. El caso era que, si el autor pensaba en un yonqui, una mamada, una puta o un fregadero, eso era exactamente lo que debía escribir. No dejarse engatusar por el preciosismo estético, la amabilidad o el ornamento, para no acabar constituyendo en tres líneas lo que podría explicarse en tres palabras.

casi todas las sinfonías y óperas podrían ser más breves.

Bukowski, Charles, “Unas observaciones sobre música” (Bukowski [2002]

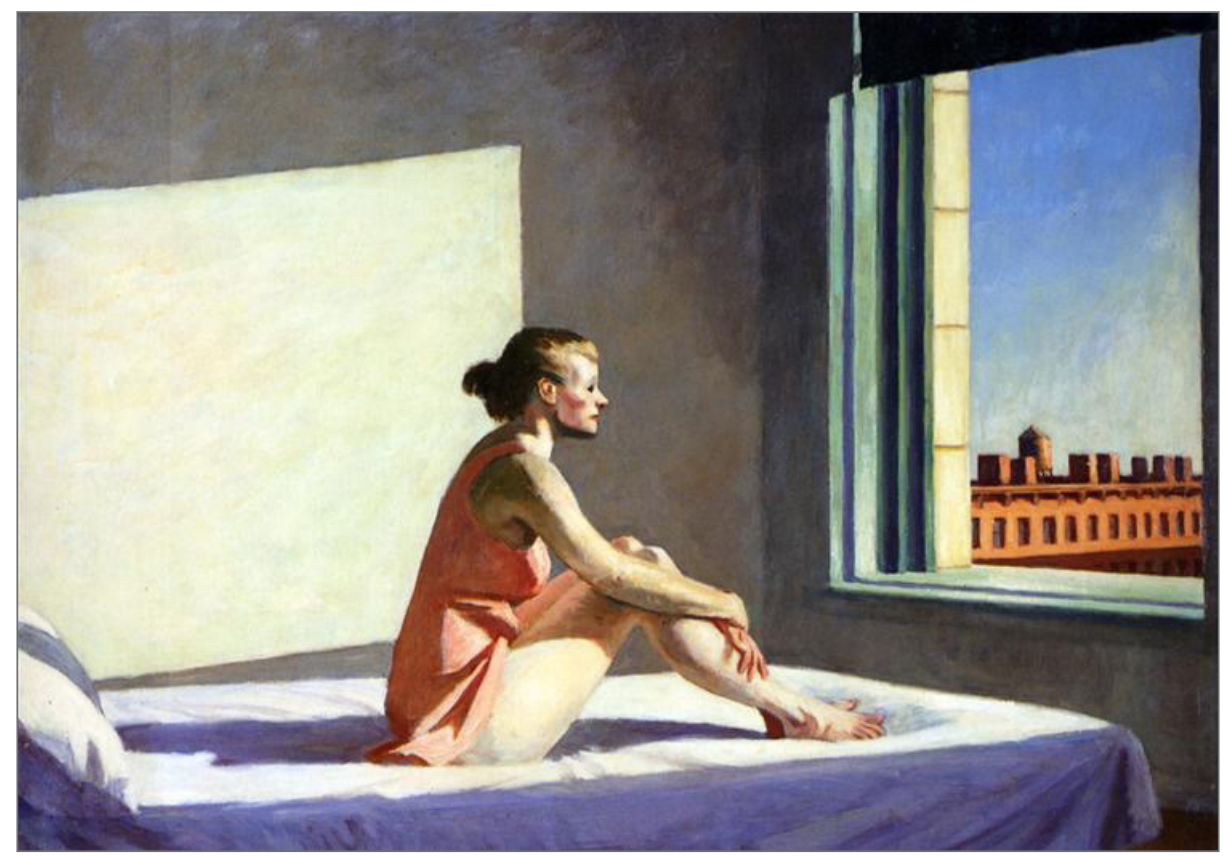

Hopper, Edward, Morning Sun, 1952. 
Esta sencillez técnica no implica sólo una estructura discursiva esencial, carente de florituras, sin el menor atisbo de musicalidad. La sencillez técnica implica historias sencillas. La concisión y la falta de heroísmos en dichas historias ofrece un genuino tono de honestidad que, claro está, si bien no suponía la única oferta cultural con un trasfondo veraz y directo, sí contaba con un desencanto particularmente incisivo y, finalmente, acabó suponiendo una postura distanciada de las demás. Igual que en un cuadro de Edward Hopper, los elementos son escasos y no cuentan nada que no se conozca de antemano. Aún así ejercen atracción.

Este lugar apartado, marginal, solitario e individual, creado por el realismo sucio, se contrapone a una sociedad cargada de agentes insistentemente simpatizantes, a un mundo regido por la militancia en grandes causas e ideologías. Frente a las consignas políticas, espirituales o artísticas, la realidad queda resumida a un individuo tratando sobrevivir, conviviendo consigo mismo.

\section{CONCLUSIONES}

El realismo sucio jugó un papel determinado en la contracultura del siglo XX al configurar una posición esencialmente individualista que influyó, distanciada de las grandes ideologías, primero en la sociedad estadounidense y, con el tiempo, a nivel internacional. Con respuestas contundentes a las problemáticas formales de la literatura, sus fundamentos se equiparan en solidez y coherencia a otras corrientes coetáneas.

En la obra de Charles Bukowski reside una definición global del movimientoy, en lo que al ámbito poético concierne, un nuevo paradigma de poeta estadounidense, alejado de los clásicos tintes populares retomados por Allen Ginsberg y otros. Dado que el realismo sucio no es un movimiento plenamente reconocido, no existe una clara adscripción a sus fundamentos por parte de artistas pertenecientes a otros campos, tal como sucede en el caso de la beat generation.

Cabe preguntarse si, ahondando en la naturaleza de esta corriente, no encontraríamos toda una serie de autores y obras que han experimentado una influencia decisiva por parte de la misma. Tal vez, atando cabos, se pueda trazar una trayectoria desde los primeros indicios del movimiento hasta el día 
de hoy y, finalmente, encontrar a los herederos del realismo sucio en el arte contemporáneo.

\section{Referencias}

Bukowski, Charles. (2002) 2005. Escrutaba la locura en busca de la palabra, el verso, la ruta: Nuevos poemas. Traducción de Eduardo Iriarte Goñi. Madrid: Visor

Ginsberg, Allen. (1956) 2006. Aullido y otros poemas. Traducción de Rodrigo Olavarría. Barcelona: Anagrama

Whitman, Walt. (1855) 2009. Hojas de hierba. Edición de Francisco Alexander. Madrid: Visor 